\title{
Behavior Study of Reinforced Concrete Frame with Different Ductility Levels under Explosive Loading
}

\author{
Waseem Haleem Al-Baghdadi and Amjad Hameed Abd-alrazaq \\ Department of Civil Engineering, Faculty of Engineering, University of Kufa, Al-Najaf, Iraq
}

\begin{abstract}
Due to increased levels of unrest and potential terrorist attacks, more studies on structures and their resistance to explosion are so imperative. Many existing structures cannot resist blast effects, therefore, improvement to their resistance to the explosive loading is required. The present study is an attempt to study the behavior of RC frames which having different levels of ductility (designed according to ABA code), these frames had been exposed to explosive loading. Taking into account the significant role of Fiber-Reinforced Plastic (FRP) in improving ductility, a comparison has been made between the behavior of high ductile frames and that of FRP. Analyses were carried out by using Abaqus/Explicit Software. It should be noted that the proposed models were verified through laboratory testing.
\end{abstract}

Key words: Explosive loading, ductility, nonlinear dynamic analysis, Reinforced Concrete (RC) frames, Fiber-Reinforced Plastic (FRP), models

\section{INTRODUCTION}

Explosions caused by bombs planted (around or inside) buildings have a devastating impacts that may damage different parts of the existing structures. Explosions apply a very large force on structures and lead to fire and loss of many of original properties for building structures.

Concrete had a compressive strength capacity at one direction while at the other direction the required strength is provided by reinforcing bars. It should be noted, however that the direction at which explosion impacts the building cannot be identified. Reinforced concrete members have showed a superior behavior, they are heavier and represent better damping and energy absorption properties. In the United States, only RC buildings can be constructed at places where explosions are most likely to occur.

\section{MATERIALS AND METHODS}

Ductility: While designing structural members within the range of elastic and linear behavior, strength is a very important standard to be considered. Nevertheless, the behavior of these structural members cannot always be limited to this range. Therefore, structural members should be allowed to experience the behaviors outside the linear elastic range where structure and structural members are examined, depending on the ductility (Mutalib and Hao, 2010).

Via. taking into account energy absorption and the degree to which material shape could be altered, ductility is determined. Materials with low energy absorption are called brittle where as those with high levels of absorption are referred to as ductile materials.

Ultimate failure in brittle materials may be occurred when the point of ultimate strength is reached as for ductile materials experience failure after absorbing considerable amount of energy (Bangash and Bangash, 2006). Energy absorption is manifested in change and can be calculated by using quantities associated with deformation. Such parameters include deflection at mid-span for beam members or moment around support for beam or planar members.

All structural materials had elastic behavior that has been simplified to facilitate required computations. The model known as perfect elasto-plasticity is the simplest plus most well-known model utilized for this purpose.

Verification of the model: Crawford and Malvar laboratory results in ACI structural had been employed to verify the outcomes. Abaqus was utilized to conduct modeling, it is a software that uses finite element analysis while the nature of explosive loadings was simulated by utilizing explicit with 3D elements for concrete models. Having utilized the embedded elements with a 3D reduced-integration eight-node host element (C3D8R), the three-dimensional model of $\mathrm{RC}$ behavior has been enhanced, on other hand, rebar network was modeled as a guest element through employing a 2-node beam (B31) (Crawford et al., 1997) (Fig. 1).

Corresponding Author: Waseem Haleem Al-Baghdadi, Department of Civil Engineering, Faculty of Engineering, 


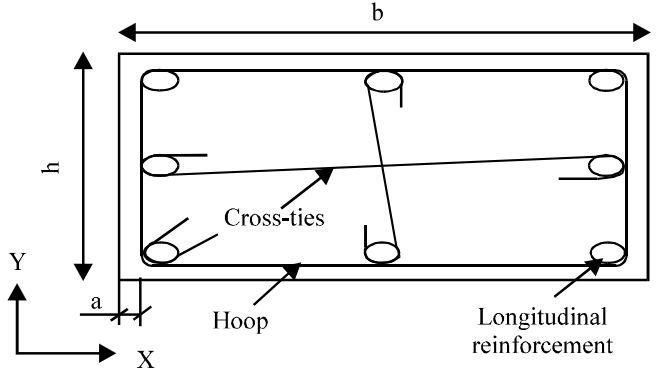

Fig. 1: Description of the column modeled in this study

Table 1: Plastic properties of steel

\begin{tabular}{lc}
\hline Stress $(\mathrm{MPa})$ & Plastic strain \\
\hline 440 & 0.00 \\
500 & 0.13 \\
\hline
\end{tabular}

Table 2: Plastic properties of (pressurized) concrete

\begin{tabular}{lll}
$\begin{array}{l}\text { Compressive } \\
\text { stress }(\mathrm{MPa})\end{array}$ & Nonlinear strain & $\begin{array}{c}\text { Compressive } \\
\text { damage parameter }\end{array}$ \\
\hline 15.19 & 0 & 0 \\
20.32 & 0.000111043 & 0.0006 \\
24.572 & 0.000167884 & 0.003 \\
30.9 & 0.00035495 & 0.009 \\
34.3 & 0.000856897 & 0.06 \\
24.36 & 0.002239064 & 0.1 \\
5.87 & 0.005984989 & 0.7 \\
2.9 & 0.008153061 & 0.9 \\
\hline
\end{tabular}

Table 3: FRP properties

\begin{tabular}{ll}
\hline Variables & Values \\
\hline Density & $1,580 \mathrm{~kg} / \mathrm{m}^{3}$ \\
Longitudinal modulus & $138 \mathrm{GPa}$ \\
Transverse modulus & $9.65 \mathrm{GPa}$ \\
Internal shear modulus & 5.24 \\
External shear modulus & 2.24 \\
Internal shear strength & $71 \mathrm{MPa}$ \\
External shear strength & $2,280 \mathrm{MPa}$ \\
Longitudinal tensile strength & $57 \mathrm{MPa}$ \\
Transverse tensile strength & $1,440 \mathrm{MPa}$ \\
Transverse compressive strength & $238 \mathrm{MPa}$ \\
\hline
\end{tabular}

Table 4: Maximum deformation of the element and comparison to laboratory tests

\begin{tabular}{lccc}
\hline Type of study & TNT (kg) & $\begin{array}{c}\text { Distance from } \\
\text { column }(\mathrm{m})\end{array}$ & $\begin{array}{c}\text { Maximum } \\
\text { deformation }(\mathrm{mm})\end{array}$ \\
\hline Laboratory (no FRP) & 682 & 6.1 & 48 \\
FEM (no FRP) & 682 & 6.1 & 47 \\
Laboratory (with FRP) & 682 & 6.1 & 18 \\
FEM (with FRP) & 682 & 6.1 & 20 \\
\hline
\end{tabular}

Material properties: Steel behavior modeling; modulus of elasticity: $200 \mathrm{GPa}$; Poisson's ratio: 0.3 (Table 1); Elastic properties of concrete; modulus of elasticity: $29 \mathrm{GPa}$; Poisson's ratio: 0.2 (Table 2 and 3).

According to Shi et al. (2010), only displacement at the middle point of the column had been mentioned, therefore, the only deformation at the middle point was analyzed here. A TNT load of $682 \mathrm{~kg}$ was applied at a distance of $6.1 \mathrm{~m}$ from the column of the first story as shown in Table 4.
Description of the model: To begin with in accordance with the construction code with three ductilities: low, medium and high. A building (with its complete structural members) was designed. The building was located at a region with high levels of seismic activity and planned in the form of a moment resisting frame. After that explosive loading was applied to two-story RC frame with two spans and with different levels of ductility. The frames had been chosen in such manner that provides the beams and columns identical geometry with square cross sections. The minimum dimension and percentage of longitudinal rebar had been elected in a way that the frame stays within the elastic range with no plastic behavior during static analysis under live and dead loads (Fig. 2).

Low-ductility frame: Wherever small deformations are expected, low ductility will be appropriate for structures. Such level of ductility is related to regions with low to average seismic risks (Fig. 3).

Medium-ductility frame: In case of structures that enter the nonlinear range in response to seismic forces, average ductility will be in need. To avoid brittle fractures, proper safety must be addressed in the cross sections of these structures (Fig. 4).

High-ductility frame: High ductility is needed in structures having structural members with ability to absorb and damp significant amounts of energy and remain steady to give the safety needed. Because of beams are more ductile than columns, a total moment capacity at beam-column junction must be greater than total moment resistance of beams along each main plate (Fig. 5).

Explosive loadings: Overpressure and duration are the two parameters involved within every explosion, loading is executed depending upon aforementioned parameters. Yet, the current study does not concentrate on loading process and effects of explosion on material behavior (Anonymous, 2008). To meet the following conditions, explosive loadings had applied, explosive loading is selected based on the type of the structure with shockwaves impacting the whole structure at a stand-off distance or range (local explosions are not considered here).

Explosive loading must not result in large deformations in the structural members of the frame modeled by the analytical software. Large deformation refer to those that destruct the structure. 


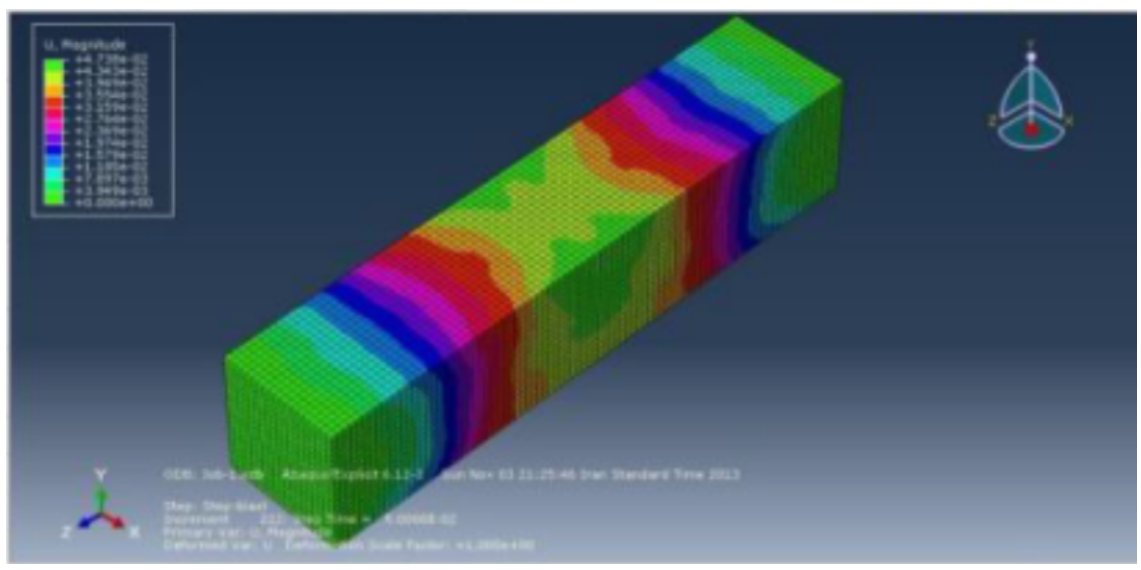

Fig. 2: Maximum deformation ( $\mathrm{mm}$ ) of the column under explosive load (no FRP used)
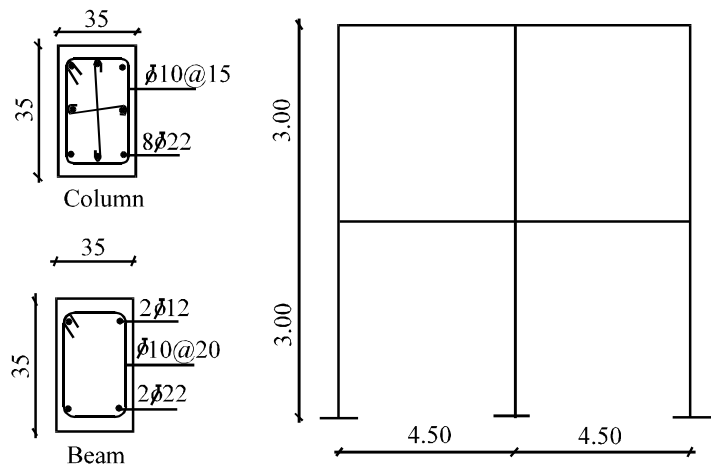

Fig. 3: Description of the column modeled in this study
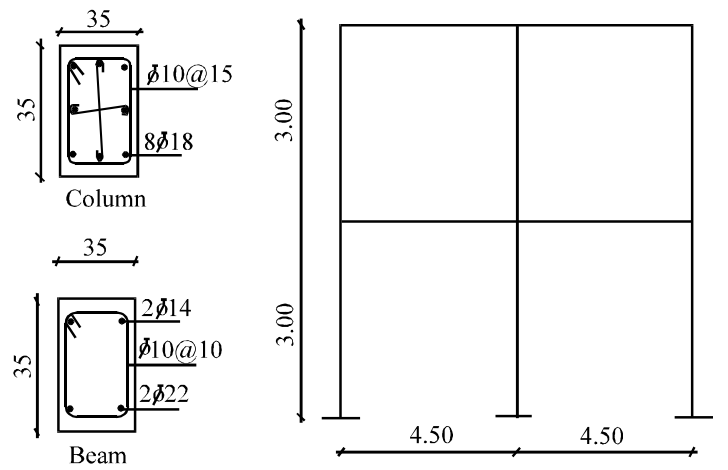

Fig. 4: Description of the column modeled in this study

Modeling: In modeling process, solid elements were utilized to model concrete frames while the reinforcement was modeled by using Wire. Explosive loading and nonlinear dynamic analysis were performed by using Abaqus Software.

Effect of source-target distance: This study deals with the comparison amid behaviors of various frames under explosive loading created by $100 \mathrm{~kg}$ of TNT at a distance of 10 and $15 \mathrm{~m}$ from the structure.
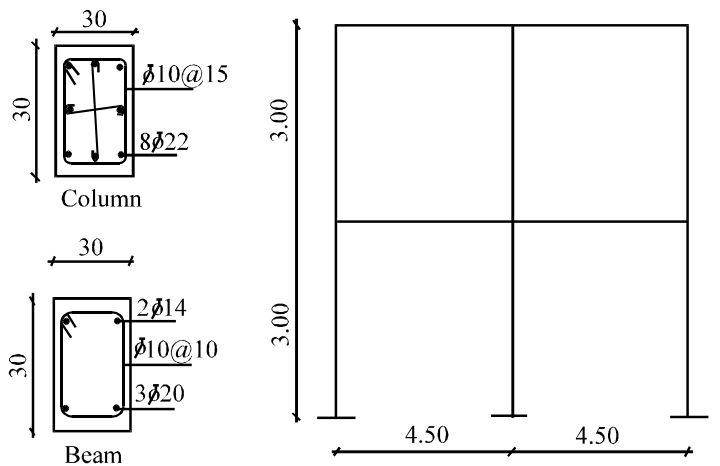

Fig. 5: Description of the column modeled in this study

- Explosive charge of $100 \mathrm{~kg}$ of TNT at $10 \mathrm{~m}$

$$
\mathrm{Z}=\frac{\mathrm{R}}{\mathrm{w}^{\frac{1}{3}}}=\frac{10 \times 3.28}{(100 \times 2.2)^{\frac{1}{3}}}=5.433 \frac{\mathrm{ft}}{\mathrm{Ib}^{3}}
$$

- Explosive charge of $100 \mathrm{~kg}$ of TNT at $15 \mathrm{~m}$

$$
\mathrm{Z}=\frac{\mathrm{R}}{\mathrm{w}^{\frac{1}{3}}}=\frac{15 \times 3.28}{(100 \times 2.2)^{\frac{1}{3}}}=8.164 \frac{\mathrm{ft}}{\mathrm{Ib}^{3}}
$$

The curves obtainable in UFC had been utilized to determine explosion parameters then explosive forces applied by $100 \mathrm{~kg}$ of TNT to the columns and ceilings at distances of 10 and $15 \mathrm{~m}$ were calculated. The following figures demonstrate the role of source-target distance.

Displacement along $\mathbf{x}$-axis: Figure $6-9$ portray displacement of concrete frame at $\mathrm{x}$ and $\mathrm{y}$ directions under explosive loading 10 and $15 \mathrm{~m}$ away from the structure, the following notes can be observed from these figures: alterations in source-target distance for frames with different levels of ductility lead to larger displacements in 


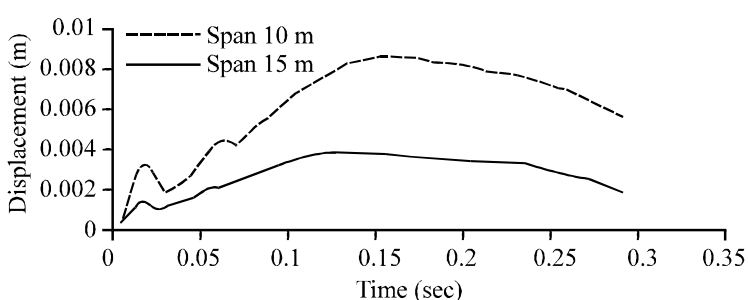

Fig. 6: Displacement (Along $\mathrm{x}$-axis) vs. time for a low-ductility frame at $10 \mathrm{~m}$ and $15 \mathrm{~m}$

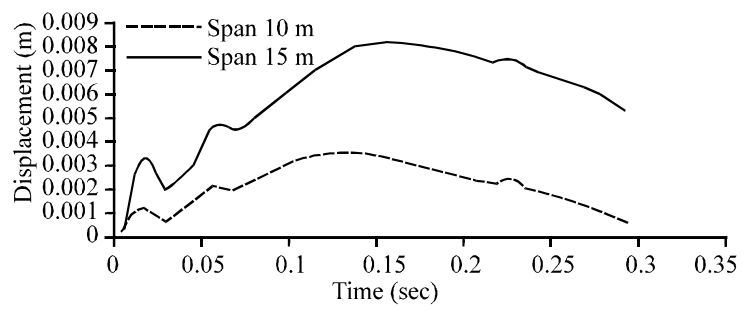

Fig. 7: Displacement (along $\mathrm{x}$-axis) vs. time for $\mathrm{a}$ medium-ductility frame

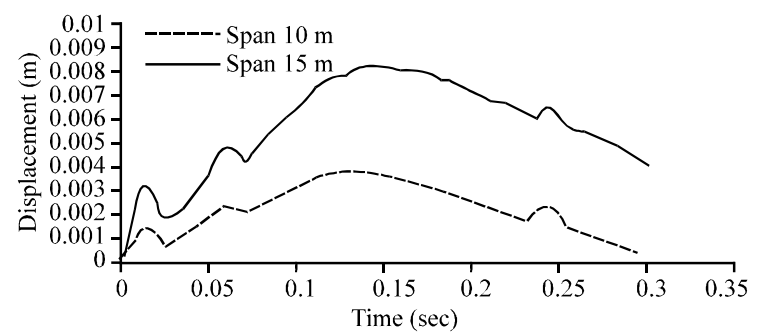

Fig. 8: Displacement (along $\mathrm{x}$-axis) vs. time for $\mathrm{a}$ high-ductility frame at 10 and $15 \mathrm{~m}$

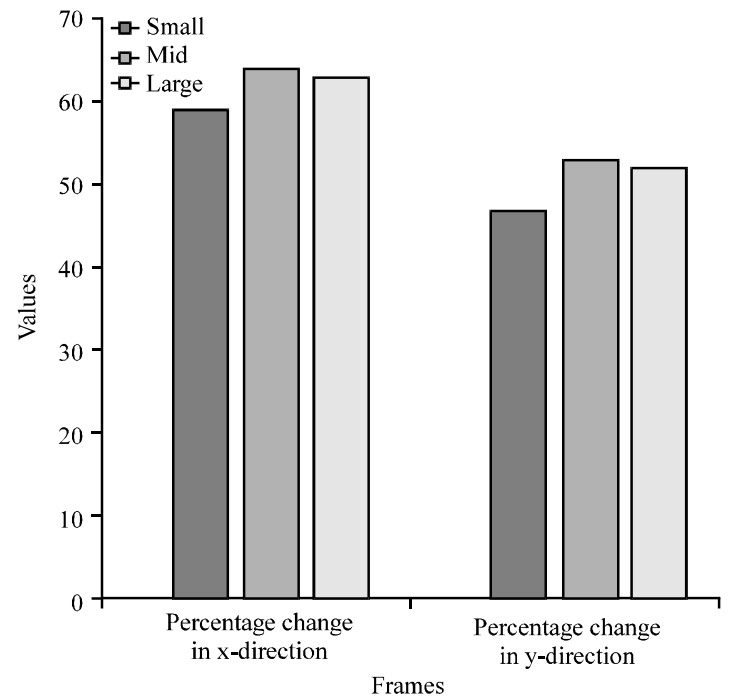

Fig. 9: Comparison of displacement of frames with different levels of ductility in $\mathrm{x}$ and $\mathrm{y}$ directions at 10 and $15 \mathrm{~m}$

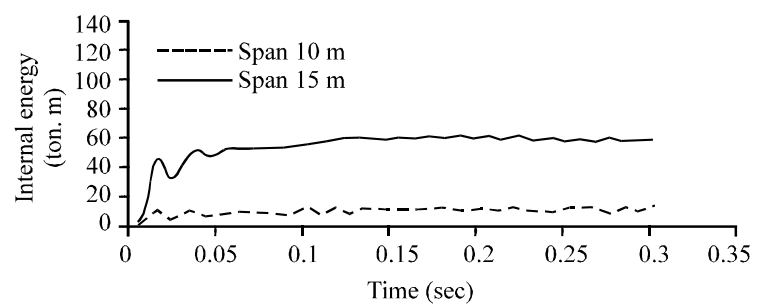

Fig. 10: Internal energy vs. time for low-ductility frame 10 and $15 \mathrm{~m}$ away from the explosion source

the direction $\mathrm{x}$ rather than in the direction $\mathrm{y}$. Displacement in both $\mathrm{x}$ and $\mathrm{y}$ directions does not alter significantly the increase of ductility.

Z-direction displacements are the same as to the ones explained above for the directions $\mathrm{x}$ and $\mathrm{y}$. However, the results for this direction have been neglected because of the required for more accurate $3 \mathrm{D}$ analysis of the structure and the need for considering diaphragm effects, etc.

Taken into account the significant variations in the amount of loading on beams and columns, it could be noticed that in all loadings, the first spot that experiences destruction under explosive loading is the two ends of the front column at the first story and, like other explosions, the damages taking place within late phases represent the results of fragments from other stories.

Internal energy: Besides other seismic parameters involved in time analysis of structures, internal energy can as well a significant element which must be considered, since, seismic performance of structures under transverse load does not only function as maximum responses (e.g., maximum acceleration, displacement and strength) but also it functions as the capability of a structure to absorb and dissipate energy. In addition, calculating internal energy has always been a vital type of analysis for energy-based design while this type of design gives a more accurate seismic recognition for designing a structure depending upon aforementioned elements as resistance or capacity. The figures present dissimilarities in internal energy in due course for two source-target distances.

As it can be noticed in Fig. 10 and 11 and Table 5. The increment in distance from 10-15 m made an equal decrease in internal energy for frames with various levels of ductility, signifying that ductility does not play a significant role in such case.

Effects of explosive charge on behavior of frames with different ductility: By drawing on the previous outcomes in this study, the change of the response of structure (at 


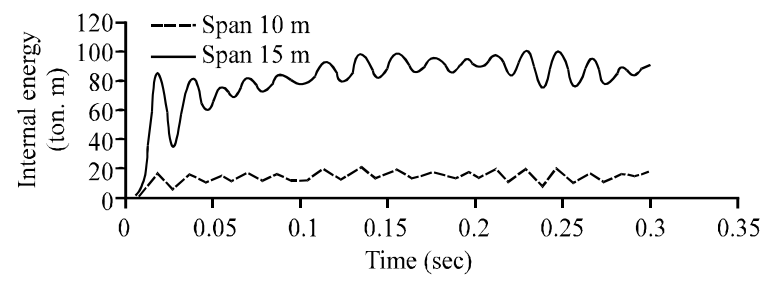

Fig. 11: Internal energy vs. time for high-ductility frame, 10 and $15 \mathrm{~m}$ away from the explosion source

Table 5: A comparison of reduction in internal energy and kinetic energy for frames with different ductility, 10 and $15 \mathrm{~m}$ from the explosion source

\begin{tabular}{lccc}
\hline Ductility & Low ductility & Medium ductility & High ductility \\
\hline $\begin{array}{l}\text { Internal energy } \\
\text { reduction (\%) }\end{array}$ & 84 & 83 & 83 \\
\hline
\end{tabular}

Table 6: Comparison of effects of changing the amount of explosive charge on variations in $\mathrm{x}$ and $\mathrm{y}$ displacements for frames with different ductility

\begin{tabular}{lccc}
\hline Ductility of frame (\%) & $\begin{array}{c}\text { Low-ductility } \\
\text { frame }\end{array}$ & $\begin{array}{c}\text { Medium-ductility } \\
\text { frame }\end{array}$ & $\begin{array}{c}\text { High-ductility } \\
\text { frame }\end{array}$ \\
\hline $\begin{array}{l}\text { Reduction in x-direction } \\
\text { displacement }\end{array}$ & 82 & 79 & 78 \\
$\begin{array}{l}\text { Reduction in y-direction } \\
\text { displacement }\end{array}$ & 97 & 92 & 89 \\
\hline
\end{tabular}

a distance of $10 \mathrm{~m}$ from the explosion source) in accordance with impacts of explosive charge via. reducing the weight of TNT from $200-100 \mathrm{~kg}$ can be detailed.

It can be noticed in Table 6, a $50 \%$ decrease in explosive charge led to an equal decrease in displacements in $\mathrm{x}$ and $\mathrm{y}$ direction for frames with various ductilities as such this signifies that ductility has no role to play in such case. Nevertheless, it must be noted that decrease in displacement is larger in y direction in comparison with $\mathrm{x}$ direction.

\section{RESULTS AND DISCUSSION}

Comparison of transverse displacement of stories for seismic and explosive loadings: The maximum transverse displacements for explosive and seismic loadings will also be compared in order to investigate the effects of explosive loadings. The results established in figure. As it can be observed the Fig. 12, seismic transverse displacement is much smaller than the displacement resulted by explosive loading with the minimum increment in displacement resulting from transverse loading on the frame with high ductility.

Based on the results indicated in the figure, it can be argued that a structure designed depending upon seismic resistance codes should be directly investigated and carefully redesigned to obtain resistance in opposition to explosive loadings. Similarly, it should be noticed that the dissimilarities between the outcomes for explosive and seismic cases have become smaller as the stand-off distance increments.

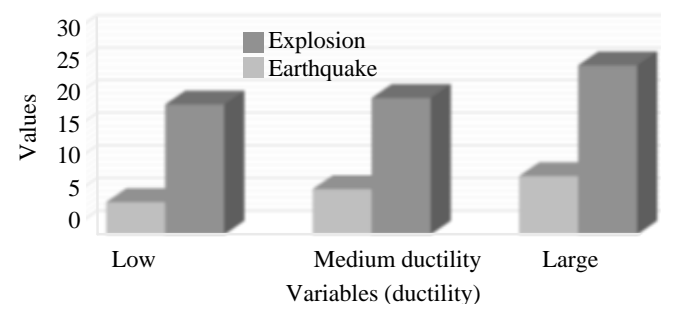

Fig. 12: Comparison of maximum transverse displacement of stories for explosive and seismic loadings

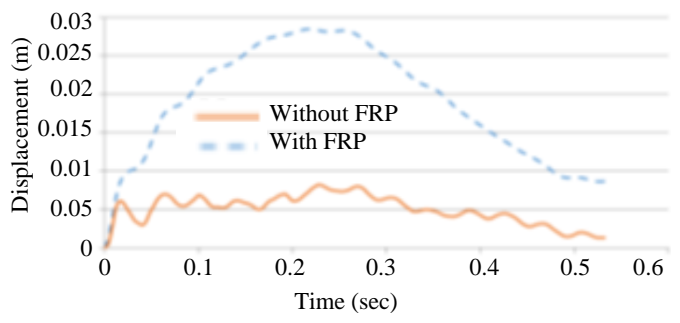

Fig. 13: Comparison of time-displacement curves for FRP and non-FRP columns under explosive loading

A several structures are not appropriately intended to withstand substantial burdens connected inside a brief time frame because of fast and capable shockwaves. Figures and results acquired from nonlinear dynamic examination of RC outlines in Abaqus unmistakably show the impact of source-target remove and in addition the kind of cement. This portrays the part of the parameter $\mathrm{Z}=\mathrm{R} / \mathrm{W}^{1 / s}$ and the relative significance of separation or range $(\mathrm{R})$ and weight of explosive charge $(\mathrm{W})$.

Application of fiber reinforced plastic in columns Displacement in FRP: Compression individuals are regularly encased to expand ductility and enhance bearing limit in districts with abnormal amounts of seismic action. Focal points of FRP enclosure incorporate averting pounding of concrete cover around longitudinal rebar, avoiding slips and clasping, upgrading compressive quality and enhancing twisting limit. FRP enclosure additionally alters malleability by expanding limit. Therefore, a FRP-fortified part won't encounter over-burdening while the casing will be shielded from sudden disappointment (Fig. 13).

Figure 13 presented that maximum displacement is $29 \mathrm{~mm}$ when no FRP is utilized whereas FRP enclosure decreases this displacement to $9 \mathrm{~mm}$.

Response to explosive loading for different cases of FRP enclosure

Case 1; Reinforcing the concreting side facing the explosive loading: The side facing explosion is subjected to reflected pressure. Reinforcing this side has 
decremented maximum deformation to about $21 \mathrm{~mm}$. This results in approximately $20 \%$ of decrease in maximum deformation. It should be noticed that this proportion points to 20 of the total $100 \%$ decrease in maximum deformation due to the use of FRP.

Case 2; Reinforcing lateral sides of concrete: Parallel sides bear littler stacking were contrasted with the side confronting blast, since, they don't encounter reflected weight and face a shockwave hosed by the front side. A decrease of around $15 \%$ in most extreme disfigurement can be accomplished by applying this kind of support.

Case 3; Reinforcing the back side of concrete (the side away from explosion): Explosive burden makes a substantial twisting on the concrete segment which thus, brings about strain on the posterior. In this way, it is critical to fortify this side. FRP sheets are known for their high rigidity and fortifying the posterior that is especially, critical in lessening most extreme displacement. This makes a commitment of around $65 \%$ to lessening in greatest displacement. A further analysis of these three cases signifies that the stronger the back side and the front side are the better the performance of concrete against explosive loads will be. Maximum displacement and stress are significantly reduced in these cases.

The increase in ultimate strain as a result of using FRP is very important in improving resistance of structures, since, it enhances ductility of structural members within the non-elastic range. A proper strengthening solution must be chosen through considering various parameters including followings.

FRP wraps (jackets) are useful when the area surrounding the columns (beams) is completely accessible if economic considerations are not of any issue. Amount of impact and cyclic loadings. The required amount of increase in shear capacity. FRP capabilities.

\section{CONCLUSION}

The present study investigated and made comparisons between the behaviors of concrete frames with various levels of ductility under explosive loading. Increased ductility results in incremented displacement in this manner decreasing maximum stress in structures. Regardless of ductility, no significant variation in displacement was observed in $\mathrm{x}$ and $\mathrm{y}$ directions when source-target distance (range) was changed. Changes in displacement as a result of changes in source-target distance were larger in $\mathrm{y}$ direction compared to displacements in $\mathrm{x}$ direction regardless of ductility of concrete frame.
Increase in the distance from $10-15 \mathrm{~m}$ results in an equal reduction in internal energy for frames with different levels of ductility, indicating that ductility does not play a significant role in this regard.

A $50 \%$ decrease in explosive charge led to an equal decrease in displacements in $\mathrm{x}$ and $\mathrm{y}$ direction for frames with various ductilities as such this signifies that ductility has no role to play in such case. Nevertheless, it must be noted that decrease in displacement is larger in y direction in comparison with $\mathrm{x}$ direction.

Seismic transverse displacement is much smaller than the displacement caused by explosive loading with the minimum increase in displacement resulting from transverse loading on the frame with high ductility.

A structure designed based on seismic resistance codes should be closely examined and carefully redesigned to achieve resistance against explosive loadings. It should be noted that the differences between the results for explosive and seismic cases become smaller as the stand-off distance increases or explosive charge decreases.

Maximum displacement in the case without utilizing FRP was about $29 \mathrm{~mm}$ while FRP reinforcement reduced this value to about $9 \mathrm{~mm}$. Given the different FRP arrangement examined here, it follows that the stronger the back side and the front side can be the better the performance of concrete against explosive loads will be. Maximum displacement and stress are significantly reduced in these cases. The increase in ultimate strain as a result of using FRP is very important in improving resistance of structures, since, it enhances ductility of structural members within the non-elastic range.

\section{REFERENCES}

Anonymous, 2008. Structure to resist the effects of accidental explosion. United States Army Corps of Engineers, Washington, D.C., USA.

Bangash, M.Y.H. and T. Bangash, 2006. Explosion-Resistant Buildings: Design, Analysis and Case Studies. Springer, Berlin, Germany, Pages: 784.

Crawford, J.E., J.L. Malvar, J.W. Wesevich, J. Valancius and A.D. Reynolds, 1997. Retrofit of reinforced concrete structures to resist blast effects. ACI. Struct. J., 94: 262-271.

Mutalib, A.A. and H. Hao, 2011. Development of P-I diagrams for FRP strengthened RC columns. Intl. J. Impact Eng., 38: 290-304.

Shi, Y., Z.X. Li and H. Hao, 2010. A new method for progressive collapse analysis of RC frames under blast loading. Eng. Struct., 32: 1691-1703. 